\title{
Void Node Detection and Packet Re-routing in Underwater Wireless Sensor Network
}

\author{
${ }^{1}$ Ekaterina Katya, ${ }^{2}$ S.R. Rahman \\ ${ }^{1}$ Professor, Department of Wireless Engineering, State University Russia \\ ${ }^{2}$ Professor, Computer Science and Engineering, State University Mexico \\ 'ekkatya1975@mail.ru
}

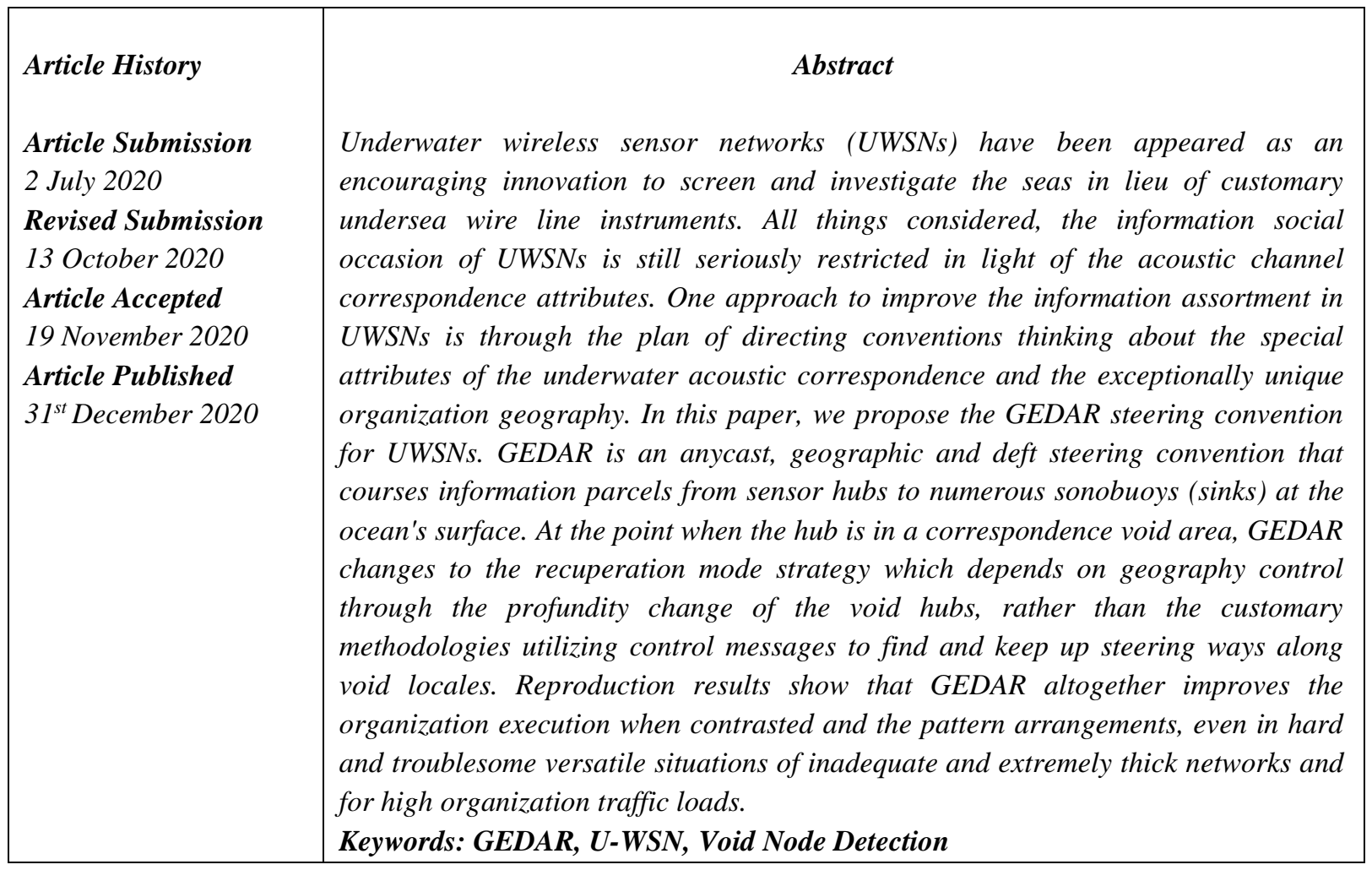

\section{Introduction}

Ocean have more than $2 / 3$ of the Earth's surface. These surroundings are critical for human existence in light of the fact that their parts on the essential worldwide favourable to diction, carbon dioxide $(\mathrm{CO} 2)$ retention and Earth's cli-mate guideline, for example. In this specific circumstance, underwater wireless sensor networks (UWSNs) have picked up the consideration of the logical and mechanical networks due their capability to screen and investigate oceanic environments. UWSNs have a wide scope of potential applications, for example, to observing of marine life, contamination content, geo-consistent cycles on the sea floor, oilfields, atmosphere, and waves and seaquakes; to gather oceanographic information, sea and seaward examining, route help, and mine acknowledgment, notwithstanding being used for strategy observation applications .Acoustic correspondence has been considered as the lone possible technique for underwater correspondence in USWNs. High recurrence radio waves are firmly invested in water and optical waves experience the ill effects of substantial dispersing and are confined to short-go view applications. By the by, the underwater acoustic channel presents enormous and variable postponement as contrasted and radio recurrence (RF) correspondence, because of the speed of sound in water that is roughly $1: 5103 \mathrm{~m} / \mathrm{s}$ (five significant degrees lower than the speed of light $(3108 \mathrm{~m} / \mathrm{s})$ ); transitory way misfortune and the high commotion bringing about a high piece blunder rate; seriously restricted data transfer capacity because of the solid constriction in the acoustic channel and multipath blurring; shadow zones; and the high correspondence energy cost, which is of the request for several watts. In this specific situation, geographic directing worldview appears to be a promising strategy for the plan of steering conventions for UWSNs. Geographic directing, additionally called of position-based steering, is 
basic and adaptable. It doesn't need the foundation or upkeep of complete courses to the objections. Additionally, there is no compelling reason to communicate steering messages to refresh directing way states. All things being equal, course choices are made locally. At each bounce, a locally ideal next-jump hub which is the neighbour closest to the objective, is chosen to keep sending the bundle. This cycle continues until the bundle arrives at its objective. Geographic steering can cooperate with crafty directing (OR) (geo-shrewd directing) to improve information conveyance and lessen the energy utilization comparative with parcel retransmissions. Utilizing astute directing worldview, every bundle is communicated to a sending set made out of neighbours. In this set, the hubs are requested by some measurement, characterizing their needs. In this way, a next-jump hub in the for-warding set that effectively got the bundle, will advance it just whether the most elevated need hubs in the set fizzled into do as such. The following jump forwarder hub will drop a booked transmission of a bundle on the off chance that it hears the transmission of that parcel by a higher need hub. In OR worldview, the bundle will be retransmitted just if none of the neighbours in the set gets it. The fundamental disservice of geo-astute directing is the correspondence void area issue. The correspondence void district issue happens at whatever point the current forwarder hub doesn't have a neighbour hub nearest to the objective than itself, i.e., the current forwarder hub is the nearest one to the objective.

GEDAR can lessen the measure of void hubs through the profundity change based void hub recuperation strategy. Consequently, GEDAR improves the bundle conveyance proportion and diminishes the start to finish delay for the basic situations of low and high densities and different organization traffic load, when contrasted and the cutting edge steering conventions and the straightforward geographic and astute directing (GOR) with no recuperation mode. This work fundamentally upgrades our past arrangements by researching the steering problem and the maximum neighbourhood issue in versatile underwater organization scenarios. Also, in this work we plan an artful steering convention to adapt to underwater acoustic communication impedances. In a static underwater sensor network situation was considered with sensor hubs appended into floats and anchors. In those arrangements, steering choices and the geography association were done in a favourable to dynamic way, before the observing stage. The commitments of this work are

i) An upgraded beaconing calculation to disseminate the area of the neighbour hubs and known sonobuoys to avoid over-burdening the acoustic channel;

ii) An anycast geo-entrepreneurial steering convention propelling the bundle, at each jump, in a coordinated path towards to the nearest sonobuoy;

iii) a novel receptive most extreme neighbourhood directing strategy dependent on the profundity change of the hubs, to improve the parcel conveyance proportion by avoid long jump ways, which can expand bundle impacts and, thus, the parcel mistake rate, start to finish postponement and energy utilization.

Additionally, this work broadens our starter arrangement in that we incorporate

1) An upgraded survey of underwater sensor network steering conventions,

2) A more point by point hypothetical system and proposed calculations portrayal,

3) More recreation results including distinctive traffic load investigation and geography related and artful directing convention related execution assessment measurements.

\section{Literature Review}

Underwater sensor nodes will discover applications in oceanographic information assortment, contamination checking, seaward investigation, catastrophe avoidance, helped route and strategic observation applications. In addition, automated or self-ruling underwater vehicles (UUVs, AUVs), outfitted with sensors, will empower the investigation of regular undersea assets and social occasion of logical information in community oriented checking missions.[1] Underwater acoustic systems administration is the empowering innovation for these applications. Underwater networks comprise of a variable number of sensors and vehicles that are sent to perform cooperative checking assignments over a given zone. In this paper, a few central key parts of underwater acoustic interchanges are researched. Various designs for two-dimensional and three-dimensional underwater sensor networks are examined, and the attributes of the underwater channel are definite. The fundamental difficulties for the advancement of productive systems administration arrangements presented by the underwater climate are definite and a cross-layer way to deal with the joining of all correspondence 
functionalities is proposed. Besides, open examination issues are talked about and conceivable arrangement approaches are delineated.

In this paper we present a novel stage for underwater sensor networks to be utilized for long haul checking of coral reefs and fisheries. The sensor network comprises of static and portable underwater sensor nodes. The nodes convey highlight point utilizing a novel rapid optical correspondence framework incorporated into the TinyOS stack, and they broadcast utilizing an acoustic convention coordinated in the TinyOS stack. The nodes have an assortment of detecting capacities, including cameras, water temperature, and weight. The portable nodes can find and drift over the static nodes for information muling, and they can perform network support capacities, for example, sending, movement, and recuperation. In this paper we portray the equipment and programming design of this underwater sensor organization. We at that point depict the optical and acoustic systems administration conventions and present trial systems administration and information gathered in a pool, in streams, and in the sea. At long last, we portray our analyses with portability for information muling in this organization.[2]

Author proposed another connection metric called standardized development (NADV) for geographic directing in multihop wireless networks. NADV chooses neighbours with the ideal compromise among nearness and connection cost. Combined with the neighbourhood next jump choice in geographic steering, NADV empowers a versatile and proficient cost-mindful directing procedure. Contingent upon the goal or message need, applications can utilize the NADV system to limit different kinds of connection cost. We present proficient techniques for connect cost assessment and perform point by point reproductions in different situations. Our outcomes show that NADV beats current plans in numerous angles: for instance, in high commotion conditions with continuous bundle misfortunes, the utilization of NADV prompts $81 \%$ higher conveyance proportion. When contrasted with incorporated steering under specific settings, geographic directing utilizing NADV discovers ways whose cost is near the ideal.[3]

In this paper, we study the geographic synergistic sending (GCF) conspire, a variation of shrewd directing, which abuses the transmission nature and spatial variety of the wireless medium to improve the bundle conveyance efficiency. We will probably completely comprehend the standards, the increases, and the trade-offs of the node coordinated effort and its related cost, hence give adroit examination and direction to the plan of more effective steering/sending conventions. We initially distinguish the upper bound of the normal bundle progression (EPA) that GCF can accomplish and demonstrate the concavity of the greatest EPA. With energy effectiveness as a significant concern, we propose another measurement, EPA per unit energy utilization, which adjusts the bundle headway, dependability and energy utilization. By utilizing the demonstrated properties, we at that point propose a productive calculation which chooses an attainable applicant set that augments this neighbourhood metric. We approve our investigation results by recreations, and legitimize the viability of the new measurement by contrasting the exhibition of GCF and those of the current geographic and astute steering plans.

Ongoing occasions, for example, the 2010 Deepwater Horizon oil slick have featured the requirement for sea checking along a particular profundity skyline. A portable underwater acoustic sensor network floating with the contamination example and answering to radio-prepared surface floats can give wide inclusion, ongoing detecting and can be sent productively. This paper explores the plausibility of such an organization application by assessing the exhibition of two ongoing weight steering conventions: Depth Based Routing and HydroCast for conveying detected information from a profundity confined layer of nodes. Past work on these conventions has just cantered around low-traffic situations with rare transmissions made by nodes all through the organization, or with just one source node. A critical commitment of this paper is an examination of the impact that current float has on systems administration. The presentation of the steering conventions over the long run is estimated, under a changed 3D Meandering Current Mobility model that considers lower current paces with expanded profundity. Results show that even in a sluggish waterfront ebb and flow, parcel conveyance in an at first thick organization gets unviable inside 3 hours of float. This work recommends controlled portability the executives be explored in future to broaden network lifetime. 


\section{Summary}

\begin{tabular}{|c|c|c|c|}
\hline Sr No. & Paper Title & Techniques & Disadvantages \\
\hline 1 & $\begin{array}{l}\text { Underwater acoustic sensor } \\
\text { networks: research challenges }\end{array}$ & $\begin{array}{l}\text { Cross layered } \\
\text { Integration } \\
\text { Approach }\end{array}$ & $\begin{array}{l}\text { Research is done on } 2 \mathrm{D} \text { and } 3 \mathrm{D} \text { UWSN } \\
\text { models but static ones. TO purely } \\
\text { understand we need to consider dynamic } \\
\text { moving node approach. }\end{array}$ \\
\hline 2 & $\begin{array}{l}\text { Data collection, storage, and } \\
\text { retrieval with an underwater } \\
\text { sensor network }\end{array}$ & $\begin{array}{l}\text { Optical and } \\
\text { acoustic network } \\
\text { model approach }\end{array}$ & $\begin{array}{l}\text { Don't provide any detailing on energy } \\
\text { efficiency or security of the network. } \\
\text { Optimal Approach is still not } \\
\text { implementable. }\end{array}$ \\
\hline 3 & $\begin{array}{l}\text { Efficient Geographic Routing in } \\
\text { Multihop Wireless Networks }\end{array}$ & NADV & $\begin{array}{l}\text { Energy Efficient is better in GCF schemes } \\
\text { as compared to NADV Approach }\end{array}$ \\
\hline 4 & $\begin{array}{l}\text { On Geographic Collaborative } \\
\text { Forwarding in Wireless Ad Hoc } \\
\text { and Sensor Networks }\end{array}$ & $\mathrm{GCF}$ & $\begin{array}{l}\text { Based on GANET with collaborative } \\
\text { forwarded with GCF approach provides } \\
\text { best results in data transfer protocol. But } \\
\text { the only issues are heavy complexity and } \\
\text { more energy requirement. }\end{array}$ \\
\hline 5 & $\begin{array}{l}\text { Performance of pressure routing in } \\
\text { drifting } 3 \mathrm{~d} \text { underwater } \\
\text { networks for deep } \\
\text { monitoring }\end{array}$ & $\begin{array}{l}\text { Depth Based } \\
\text { Routing and } \\
\text { HydroCast }\end{array}$ & $\begin{array}{l}\text { Many different protocols are compared for } \\
\text { 3D UWSN. AODV works better in this } \\
\text { approach. }\end{array}$ \\
\hline
\end{tabular}

\section{Proposed Methodology}

GEDAR is an anycast, geographic and artful convention that attempts to convey a bundle from a source node to some sonobuoys. During the course, GEDAR utilizes the ravenous sending system to propel the parcel, at each hop, towards the surface sonobuoys. A recuperation mode technique dependent on the profundity change of the void node is utilized to course information parcel when it stall out at a void node. The proposed directing convention utilizes the insatiable for-warding procedure by methods for the position data of the current forwarder node, its neighbours, and the known sonobuoys, to decide the certified neighbours to keep sending the parcel towards some sonobuoys. Despite insatiable sending methodology being a notable and utilized next-jump forwarder determination system, GEDAR considers the anycast idea of underwater directing when numerous surface sonobuoys are utilized as sink nodes.

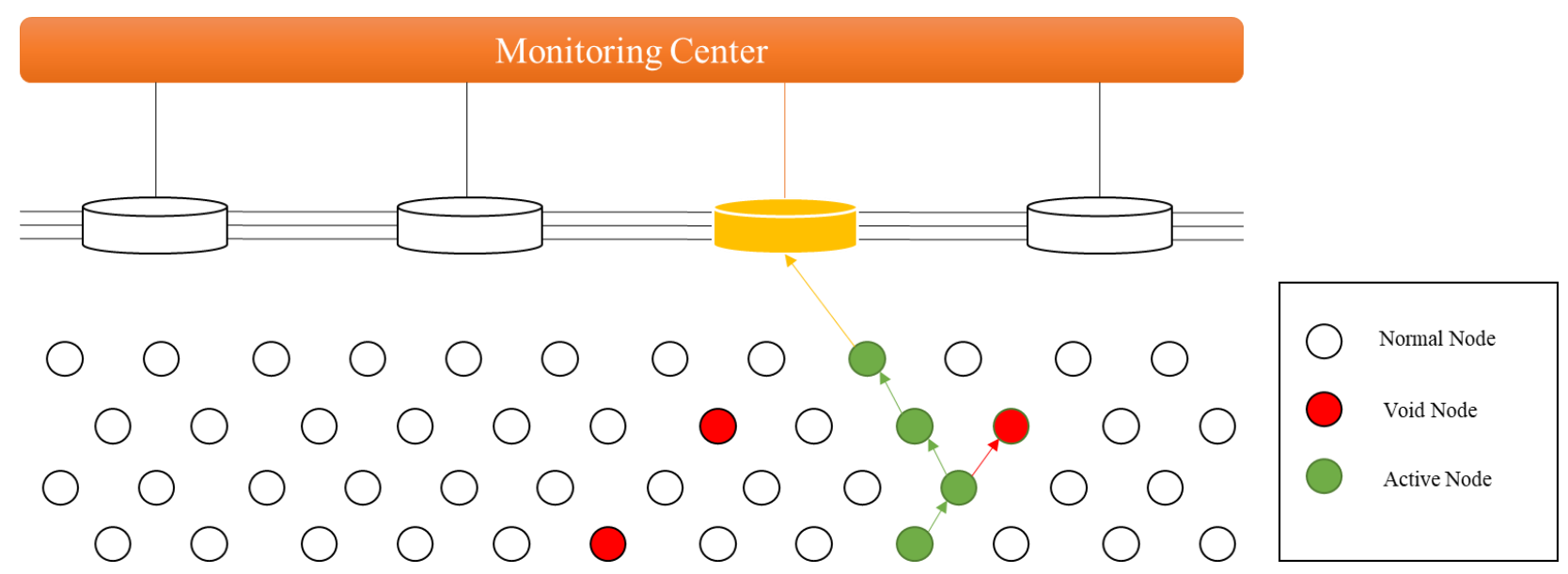

Fig 1. Proposed System Architecture 


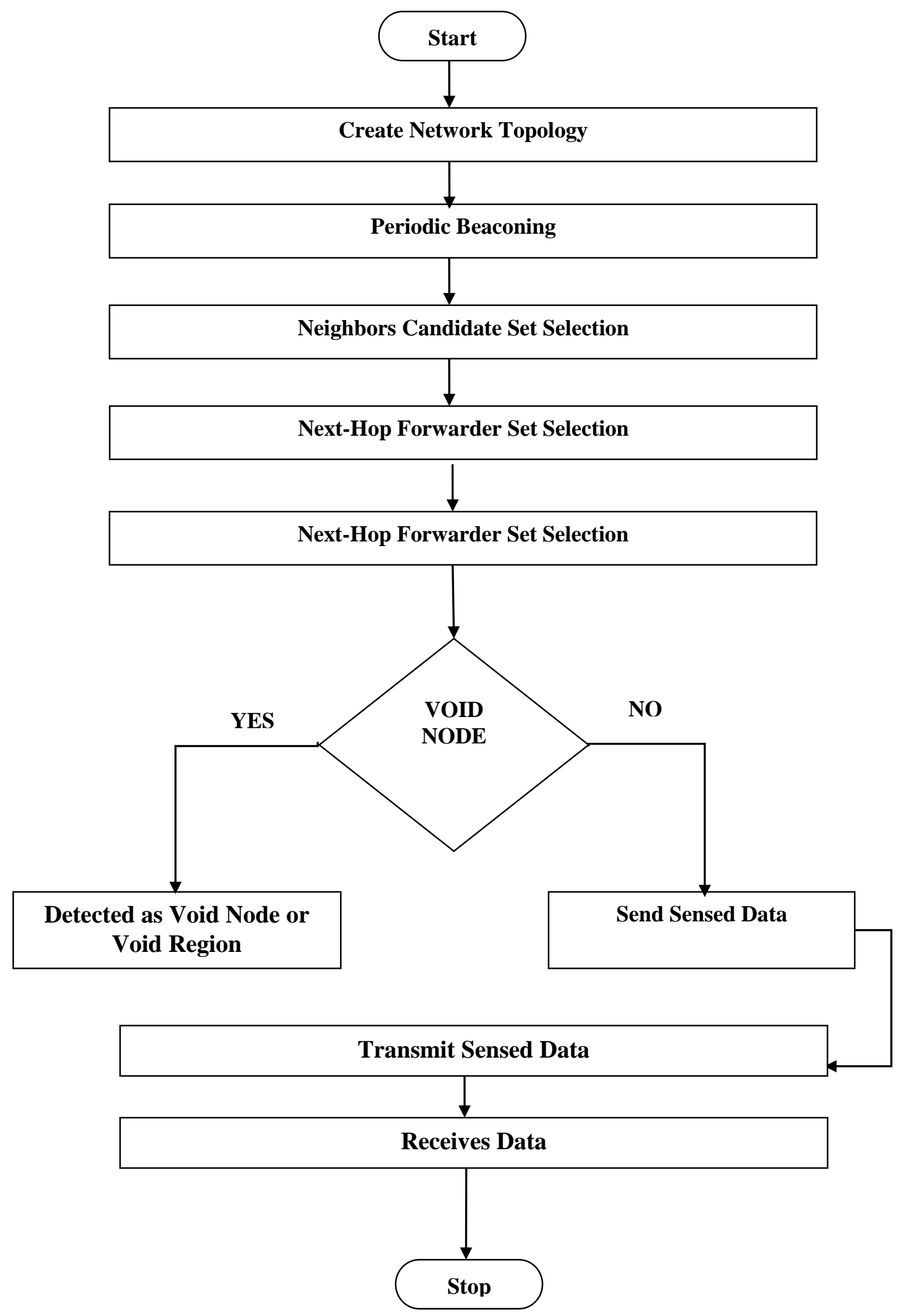

Fig 2. Basic Flow of System 


\section{Topology Creation}

In our simulations, the 32 number of sensor nodes are sent and the quantity of sonobuoys is 6 . They are arbitrarily conveyed in a district the size of 2265 X 1000. In every sensor, information parcels are produced by a Poisson cycle with a similar boundary to exceptionally low traffic load; to recreate a portable organization situation, thinks about the impact of wandering sub-surface ebbs and flows (or fly streams) and vertices. We set the principle stream speed range from $\max 5 \mathrm{~m} / \mathrm{s}$ to $\min 2.70 \mathrm{~m} / \mathrm{s}$. the nodes have a transmission range (TR) of $250 \mathrm{~m}$ and an information pace of $50 \mathrm{kbps}$. The size of the bundle is deflect mined by the size of the information payload and by the space needed to incorporate the data of the following bounce for-corrections officer set. We consider that information bundles have a payload of 150 bytes.

\section{Enhanced Beaconing}

Intermittent beaconing assumes a significant part in GEDAR. It is through occasional beaconing that every hub gets the area data of its neighbors and reachable sonobuoys, where every hub can be educated already concerning the area of all sonobuoys (as long haul submerged observing design is shaped by static nodes joined to floats as well as anchors), we need a proficient beaconing calculation that keeps the size of the intermittent guide messages short as could be expected under the circumstances. For example, if every hub ni implants its known sonobuoy areas along with its area, the size of its guide message in the most pessimistic scenario, without considering lower layer headers, bits, where $\mathrm{m}$ and $\mathrm{n}$ are the size of the grouping number and ID fields, and each geographic directions, individually. Given that the transmission of huge bundles in the submerged acoustic channel is unreasonable, we propose an improved reference point calculation that mulls over this issue. Likewise, every sensor hub installs arrangement number, it's one of a kind ID and $\mathrm{X}, \mathrm{Y}$, and $\mathrm{Z}$ position data. Also the reference point message of every sensor hub is enlarged with the data of its known sonobuoys from its set .Each hub incorporates the grouping number, ID, and the X, Y area of the its known sonobuoys. The objective is for the neighboring nodes to have the area data of the all reachable sonobuoys. GPS can't be utilized by submerged sensor nodes to decide their areas given that the high recurrence signal is quickly retained and can't arrive at nodes even limited at a few meters beneath the surface. Subsequently, every sensor hub knows its area through restriction administrations. Confinement administrations bring about extra expenses in the organization. Notwithstanding, the information with respect to the area of sensor nodes can dispose of the huge number of transmission or multicast inquiries that prompts pointless organization flooding that lessens the organization throughput. Likewise, the area data is needed to label the gathered information, track submerged nodes and targets, and to arrange the movement of a gathering of nodes.In request to stay away from long sizes of reference point messages, a sensor hub incorporates just the position data of the sonobuoys it has not scattered in the archetype round (lines 5-12). At whatever point a hub gets another guide message, in the event that it has come from a sonobuoy, the hub refreshes the relating section in the known sonobuoy set (line 20). Else, it refreshes its known sonobuoys $|\mathrm{Si}|$ set in the relating passages if the data area contained in the signal message is later than the area data in its set Si. For each refreshed passage, the hub changes the proper banner $\mathrm{L}$ to zero, showing that this data was not engendered to its neighbours (line 25). Accordingly, in the following signal message, just the passages in which the $\mathrm{L}$ is equivalent to zero are installed (lines 7-10). We add irregular butterflies somewhere in the range of 0 and 1 during the transmission of signal messages, to smaller than normal the possibility of the two crashes and synchronization. Besides, after a hub communicates a guide, it sets up another break for the following beaconing.

\section{Neighbors Candidate Set Selection}

At whatever point a sensor hub has a packet to send, it ought to figure out which neighbors are able to be the following bounce forwarder. GEDAR utilizes the insatiable sending methodology to decide the arrangement of neighbors ready to proceed with the sending towards separate sonobuoys. The essential thought of the ravenous sending technique is, in each jump, to advance the packet towards some surface sonobuoy.The neighbor up-andcomer set is resolved as follows. Leave ni alone a hub that has a packet to convey, let its arrangement of neighbors be and the arrangement of known sonobuoys at time t. 
We utilize the packet advancement (ADV) metric to prevent mine the neighbors ready to advance the packet towards some objective. The packet advancement is characterized as the distance between the source hub $\mathrm{S}$ and the objective hub $\mathrm{D}$ less the distance between the neighbor $\mathrm{X}$ and D.Thus, the neighbors up-and-comer set in GEDAR is given as:

$$
\mathcal{C}_{i}=\left\{n_{k} \in N_{i}(t): \exists s_{v} \in S_{i}(t) \mid D\left(n_{i}, s_{i}^{*}\right)-D\left(n_{k}, s_{v}\right)>0\right\},
$$

Where $\mathrm{D}(\mathrm{a}, \mathrm{b})$ is the euclidean distance between the nodes a and $\mathrm{b}$ and $s_{i}^{*} \in S_{i}(t)$,is closest sonobuoy of ni as:

$$
s_{i}^{*}=\operatorname{argmin}_{\forall s_{j} \in S_{i}(t)}\left\{D\left(n_{i}, s_{j}\right)\right\} .
$$

\section{Next-Hop Forwarder Set Selection}

GEDAR uses opportunistic routing to deal with under-water acoustic channel characteristics. In traditional multihop routing paradigm, only one neighbor is selected to act as a next-hop forwarder. If the link to this neighbor is not performing well, a packet may be lost even though other neighbor may have overheard it. In opportunistic routing, taking advantage of the shared transmission medium, each packet is broadcast to a forwarding set composed of several neighbors. The packet will be retransmitted only if none of the neighbors in the set receive it. Opportunistic routing has advantages and dis-advantages that impact on the network performance. OR reduces the number of possible retransmissions, the energy cost involved in those retransmissions, and help to decrease the amount of possible collisions. However, as the neighboring nodes should wait for the time needed to the packet reaches the furthest node in the forwarding set, OR leads to a high end-to-end latency.

For each transmission, a next-hop forwarder set $\mathrm{F}$ is determined. The next-hop forwarder set is composed of the most suitable nodes from the next-hop candidate set $\mathrm{Ci}$ so that all selected nodes must hear the transmission of each other aiming to avoid the hidden terminal prob-lem. The problem of finding a subset of nodes, in which each one can hear the transmission of all nodes, is a vari-antof the maximum clique problem,that is computationally hard. We use normalized advance (NADV) to measure the "goodness"of each next-hop candidate node in Ci.NADV corresponds the optimal trade-off between the proximity and link cost to determine the priorities of the candidate nodes. This is necessary because the greater the packet advancement is, the greater the neighbor priority becomes. However, due to the underwater channel fad-ing, the further the distance is from the neighbor, the higher the signal attenuation becomes as well as the like-lihood of packet loss.

\section{Recovery Mode}

Void node recovery procedure is used when the node fails to forward data packets using the greedy forwarding strat-egy. Instead of message-based void node recovery proce-dures, GEDAR takes advantage of the already available node depth adjustment technology to move void nodes for new depths trying to resume the greedy forwarding. We advocate that depth-adjustment based topology control for void node recovery is more effective in terms of data deliv-ery and energy consumption than message-based void node recovery procedures in UWSNs given the harsh environment and the expensive energy consumption of data communication. The GEDAR depth-adjustment based topology control for a void node recovery procedure can be briefly described as follows. During the transmissions, each node locally determines if it is in a communication void region by exam-ining its neighborhood. If the node is in a communication void region, that is, if it does not have any neighbor leading to a positive progress towards some surface sonobuoy $\left(\mathrm{C}^{1 / 4} ;\right)$, it announces its condition to the neighborhood and waits the location information of two hop nodes in order to decide which new depth it should move into and the greedy forwarding strategy can then be resumed. After, the void node determines a new depth based on two-hop connectiv-ity such that it can resume the greedy forwarding.

\section{Performance Evaluation}

In this section, evaluate the performance of simulation. We are using the xgraph for evaluate the performance. We choose the some evaluation metrics: Packet delivery ratio - the ratio of the total number of packets received 
by the destination node to the number of packet sent by the source, End-to-End delay - the time taken to be data transmitted from source node to destination node. And calculate the Energy consumption by the sensor node. Along these evaluation metrics we have to evaluate the simulation performance in xgraph.

\section{Result Analysis}

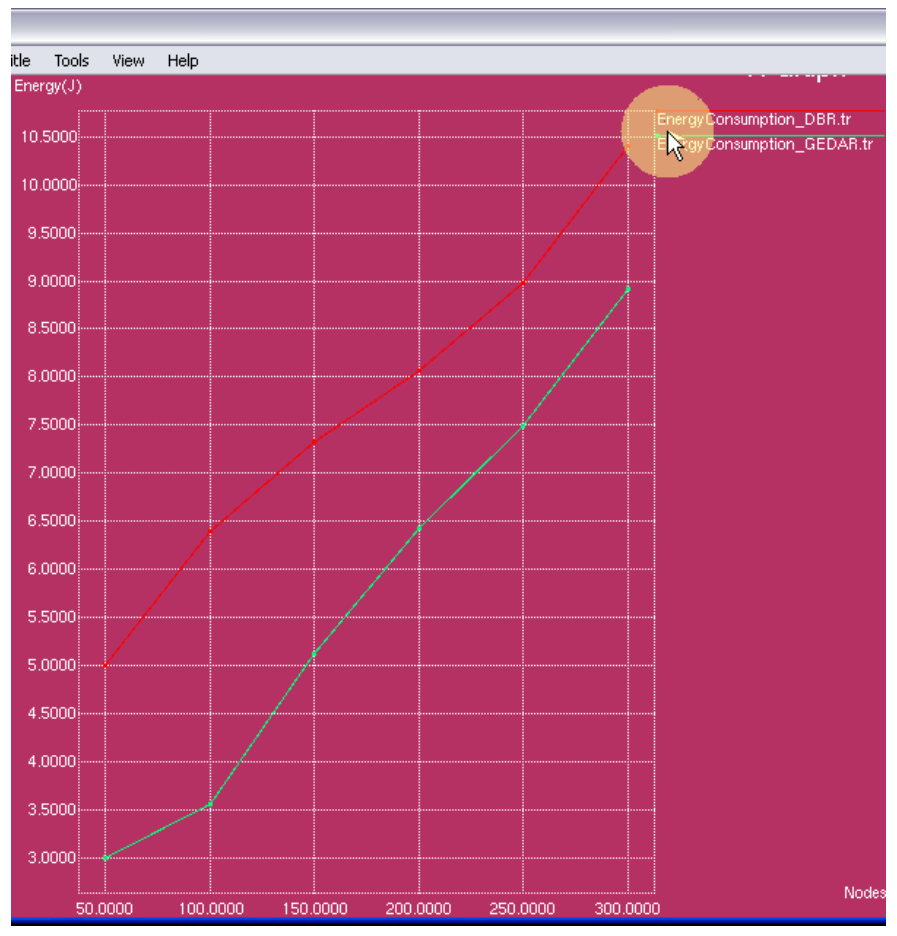

Fig 3. Energy Efficiency Comparison

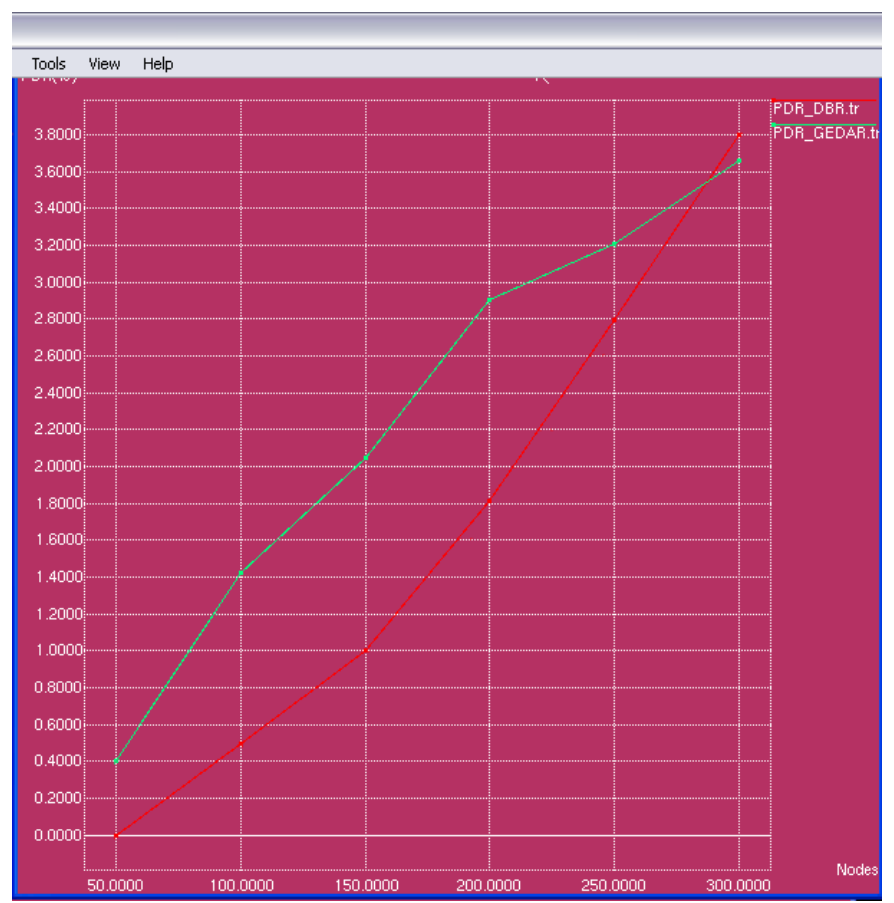

Fig 4. Packet Data Rate Comparison 


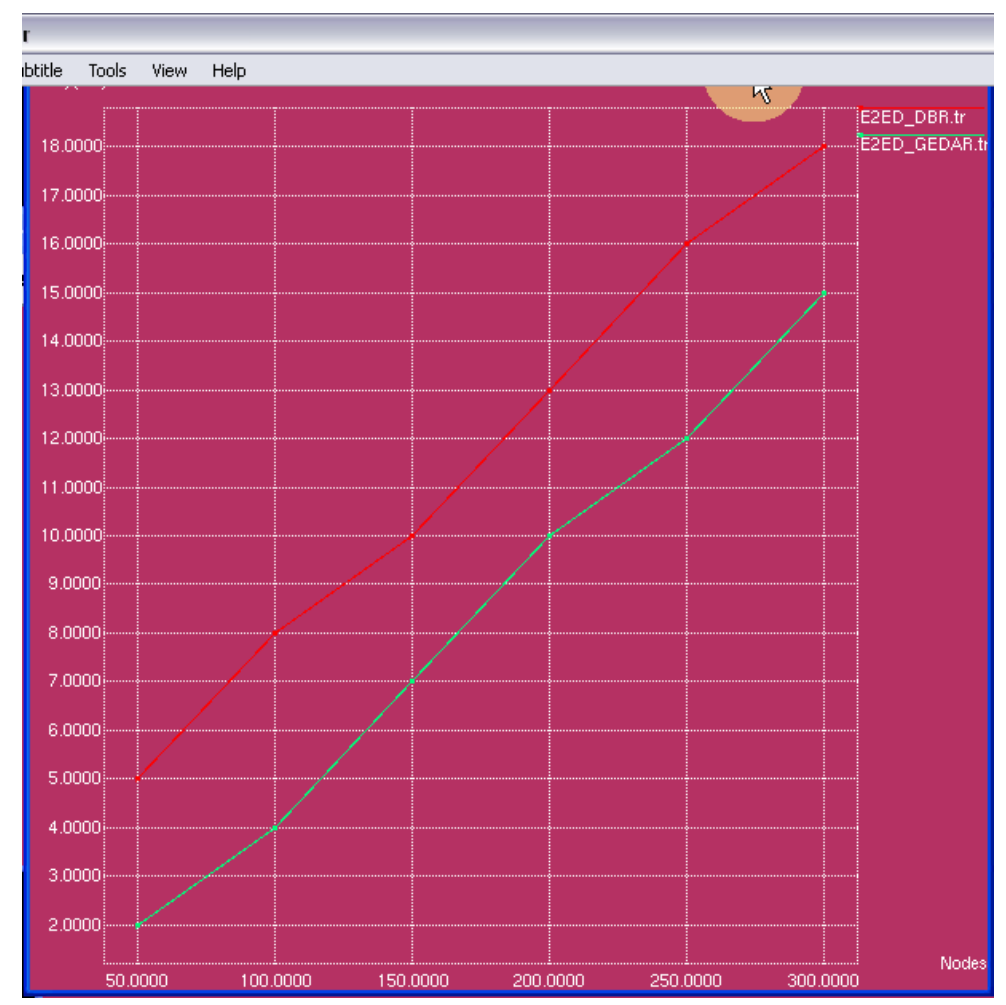

Fig 5. Energy and Data Comparison Graph

\section{Conclusion}

We proposed and assessed the GEDAR steering convention to improve the information directing in submerged sensor organizations. GEDAR is a basic and adaptable geographic steering convention that utilizes the position data of the nodes and exploits the transmission correspondence medium to avariciously and shrewdly forward information packets towards the ocean surface sonobuoys. Besides, GEDAR gives a novel profundity change based geography control instrument used to move void nodes to new profundities to defeat the correspondence void locales. Our recreation results demonstrated that geographic steering conventions dependent on the position area of the nodes are more proficient than pressure directing conventions. Additionally, astute steering demonstrated urgent for the presentation of the organization other than the quantity of transmissions needed to convey the packet. The utilization of hub profundity acclimation to adapt to correspondence void districts improved altogether the organization execution. GEDAR proficiently diminishes the level of nodes in correspondence void districts to 58 percent for medium thickness situations as contrasted and GUF and lessens these nodes to roughly 44 percent as contrasted and GOR. Thusly, GEDAR improves the organization execution when contrasted and existing submerged defeating conventions for various situations of organization thickness furthermore, traffic load. As future work, we intend to apply this geography control of profundity change standards to the plan of sharp steering conventions for UWSNs, considering diverse QoS prerequisites for information conveyance, the expense for arrives at a neighbor hub, and the lifetime of the organization.

\section{References}

[1] F. Akyildiz, D. Pompili, and T. Melodia, "Underwater acoustic sensor networks: Research challenges,"Ad Hoc Netw., vol. 3, no. 3, pp. 257-279, 2005.

[2] Vasilescu, K. Kotay, D. Rus, M. Dunbabin, and P. Corke, "Data collection, storage, and retrieval with an underwater sensor network," inProc. 3rd ACM Int. Conf. Embedded Netw. Sensor Syst., 2005, pp. 154-165.

[3] J. Partan, J. Kurose, and B. N. Levine, “A survey of practical issues in underwater networks," inProc. 1st ACM Int. Workshop Underwa-ter Netw., 2006, pp. 17-24. 
[4] J. Heidemann, M. Stojanovic, and M. Zorzi, "Underwater sensor networks: Applications, advances and challenges,”Philos. Trans. Roy. Soc. A: Math., Phys. Eng. Sci., vol. 370, no. 1958, pp. 158-175, 2012.

[5] M. Stojanovic and J. Preisig, "Underwater acoustic communi-cation channels: Propagation models and statistical character-ization," IEEE Commun. Mag., vol. 47, no. 1, pp. 84-89, Jan. 2009.

[6] P. Xie, J.-H. Cui, and L. Lao, "VBF: Vector-based forwarding pro-tocol for underwater sensor networks," in Proc. 5th Int. IFIP-TC6 Conf. Netw. Technol., Services, Protocols, 2006, pp. 1216-1221.

[7] H. Yan, Z. J. Shi, and J.-H. Cui, "DBR: Depth-based routing for underwater sensor networks," inProc. 7th Int. IFIP-TC6 Netw. Conf. Ad Hoc Sensor Netw., Wireless Netw., Next Generation Internet, 2008, pp. 72-86.

[8] U. Lee, P. Wang, Y. Noh, L. F. M. Vieira, M. Gerla, and J.-H. Cui, "Pressure routing for underwater sensor networks," inProc. IEEE INFOCOM, 2010, pp. 1-9.

[9] Y. Noh, U. Lee, P. Wang, B. S. C. Choi, and M. Gerla, "VAPR: Void-aware pressure routing for underwater sensor networks," IEEE Trans. Mobile Comput., vol. 12, no. 5, pp. 895-908, May 2013.

[10] D. Chen and P. Varshney, "A survey of void handling techniques for geographic routing in wireless networks," IEEE Commun. Surveys Tuts., vol. 9, no. 1, pp. 50-67, First Quarter 2007.

[11] F. Kuhn, R. Wattenhofer, and A. Zollinger, "Worst-case optimal and average-case efficient geometric ad-hoc routing," inProc. 4 $^{\text {th }}$ ACM Int. Symp. Mobile Ad Hoc Netw. Comput., 2003, pp. 267-278.

[12] R. W. L. Coutinho, L. F. M. Vieira, and A. A. F. Loureiro, "DCR: Depth-controlled routing protocol for underwater sensor networks," in Proc. IEEE Symp. Comput. Commun., 2013, pp. 453-458.

[13] R. W. Coutinho, L. F. Vieira, and A. A. Loureiro, "Movement assisted-topology control and geographic routing protocol for underwater sensor networks," inProc. 6th ACM Int. Conf. Model., Anal. Simul. Wireless Mobile Syst., 2013, pp. 189-196.

[14] R. W. L. Coutinho, A. Boukerche, L. F. M. Vieira, and A. A. Loureiro, "GEDAR: Geographic and opportunistic routing proto-col with depth adjustment for mobile underwater sensor networks," inProc. IEEE Int. Conf. Commun., 2014, pp. 251-256. 